\title{
Coexistence of Superconductivity and Charge Density Waves in Tantalum Disulfide: Experiment and Theory
}

\author{
Y. Kvashnin,,${ }^{1}$ D. VanGennep, ${ }^{2}$ M. Mito, ${ }^{3}$ S. A. Medvedev, ${ }^{4}$ R. Thiyagarajan, ${ }^{5}$ O. Karis, ${ }^{1}$ \\ A. N. Vasiliev, ${ }^{6,7,9}$ O. Eriksson, ${ }^{1,8}$ and M. Abdel-Hafiez,2,* \\ ${ }^{1}$ Uppsala University, Department of Physics and Astronomy, Box 516, SE-751 20 Uppsala, Sweden \\ ${ }^{2}$ Lyman Laboratory of Physics, Harvard University, Cambridge, Massachusetts 02138, USA \\ ${ }^{3}$ Graduate School of Engineering, Kyushu Institute of Technology, Fukuoka 804-8550, Japan \\ ${ }^{4}$ Max Planck Institute for Chemical Physics of Solids, D-01187 Dresden, Germany \\ ${ }^{5}$ Institut für Festkörper- und Materialphysik, Technische Universität Dresden, 01069 Dresden, Germany \\ ${ }^{6}$ Ural Federal University, Yekaterinburg 620002, Russia \\ ${ }^{7}$ Lomonosov Moscow State University, Moscow 119991, Russia \\ ${ }^{8}$ School of Science and Technology, Örebro University, SE-701 82 Örebro, Sweden \\ ${ }^{9}$ National Research South Ural State University, Chelyabinsk 454080, Russia
}

(Received 20 April 2020; revised 18 August 2020; accepted 25 September 2020; published 27 October 2020)

\begin{abstract}
The coexistence of charge density wave (CDW) and superconductivity in tantalum disulfide $\left(2 \mathrm{H}-\mathrm{TaS}_{2}\right)$ at low temperature is boosted by applying hydrostatic pressures to study both vibrational and magnetic transport properties. Around $P_{c}$, we observe a superconducting dome with a maximum superconducting transition temperature $T_{c}=9.1 \mathrm{~K}$. First-principles calculations of the electronic structure predict that, under ambient conditions, the undistorted structure is characterized by a phonon instability at finite momentum close to the experimental CDW wave vector. Upon compression, this instability is found to disappear, indicating the suppression of CDW order. The calculations reveal an electronic topological transition (ETT), which occurs before the suppression of the phonon instability, suggesting that the ETT alone is not directly causing the structural change in the system. The temperature dependence of the first vortex penetration field has been experimentally obtained by two independent methods. While a $d$ wave and single-gap BCS prediction cannot describe the lower critical field $H_{c 1}$ data, the temperature dependence of the $H_{c 1}$ can be well described by a single-gap anisotropic $s$-wave order parameter.
\end{abstract}

DOI: 10.1103/PhysRevLett.125.186401

Coexistence of superconductivity with competing physical phenomena such as magnetic or charge order has been of interest for the condensed matter community for a long time [1-3]. A commonly accepted argument says that for the materials exhibiting competing ground states, suppressing the magnetic or charge order helps to stabilize the superconducting (SC) phase. This is the case, for instance, in layered materials that are composed of two-dimensional (2D) building blocks, with periodic modulations of the charge carrier density, so-called charge density waves (CDWs) [4-6]. Classic examples are the members of the transition-metal dichalcogenide family (TMDs) $\mathrm{MX}_{2}$, where $M=\mathrm{Nb}, \mathrm{Ti}$, Ta, Mo and $X=\mathrm{S}$, Se. TMDs provide an ideal playground for studying semiconductors, metals, and superconductors in 2D using the same structural template [7-10]. At ambient pressure and without intercalation or chemical

Published by the American Physical Society under the terms of the Creative Commons Attribution 4.0 International license. Further distribution of this work must maintain attribution to the author(s) and the published article's title, journal citation, and DOI. Funded by Bibsam. substitution, $2 \mathrm{H}-\mathrm{TaS}_{2}$, a prominent member of the vast family of TMDs, exhibits both superconductivity and a canonical CDW phase transition whose mechanisms remain controversial, even after decades of research [11-13]. Despite extensive studies, the current understanding of the microscopic origin of the $\mathrm{SC}$ mechanism and the $\mathrm{CDW}$ state is not complete. The $\mathrm{SC}$ transition temperature $\left(T_{c}\right)$ increases while the CDW lock-in temperature falls down with chemical doping [13], increasing thickness of the sample [14], and external pressure [15-17]. Several theoretical mechanisms behind the formation of CDW have been proposed [18]. For TMDs, the following origins were extensively discussed: Fermi surface nesting [19], saddle points near Fermi surface [20], exciton-phonon [21], or electron-phonon coupling [22-26]. The most recent experimental evidence suggests that the latter plays a decisive role for CDW stabilization in Ta systems [27,28]. It is thus of profound importance to understand the interplay between electronic and crystal structure in $2 \mathrm{H}-\mathrm{TaS}_{2}$. Additionally, there is no general consensus on the origin of SC pairing mechanisms in this material and further studies are necessary to elucidate this issue. 
Whatever the proposed understanding of the relation between CDW and superconductivity is, it is important to determine the exact dependence of $T_{c}$ and the CDW phase with pressure [11]. Within this scope, through combined complementary experimental techniques supplemented with theoretical calculations on $2 \mathrm{H}-\mathrm{TaS}_{2}$ [29], we derive a previously not discussed pressure-temperature phase diagram. We explore external pressure as a tool to tune the phonon dispersions and thus the stability of the CDW phase. Pressure is considered a very clean way to tune basic electronic and structural properties without changing the stoichiometry of a material [30,31]. Our analysis shows that the temperature dependence of the lower critical fields, $H_{c 1}(T)$, is inconsistent with a simple isotropic $s$-wave type of the order parameter but are rather in favor of the presence of an anisotropic $s$ wave. These observations clearly show that the $\mathrm{SC}$ energy gap in $2 \mathrm{H}-\mathrm{TaS}_{2}$ is nodeless.
Raman response of $2 \mathrm{H}-\mathrm{TaS}_{2}$ at ambient pressure and room temperature is presented in Fig. 1(a), where three regular phonons are observed: (i) Two-phonon process at $180.3 \mathrm{~cm}^{-1}$; (ii) $E_{2 g}$, an in-plane vibrational mode at $288.1 \mathrm{~cm}^{-1}$; and (iii) $A_{1 g}$ the out-of-plane mode at $405.4 \mathrm{~cm}^{-1}$. These values agree well with the reported works [32,33]. Figure 1(b) shows the Raman spectra under hydrostatic pressure up to $40 \mathrm{GPa}$. The two-phonon mode originates from second order scattering of acoustic and quasiacoustic momentum phonons near the CDW wave vector. By application of pressure, all Raman modes show decreases in intensity, the peaks become wider, and the positions show blueshifts. Moreover, we observed a splitting of $E_{2 g}$ peak at $20.2 \mathrm{GPa}$ which may be due to the pressure-induced structural phase transition in $2 \mathrm{H}-\mathrm{TaS}_{2}$. The positions of the three peaks vs pressure are shown in Fig. 1(c). The Raman mode at $180.3 \mathrm{~cm}^{-1}$, which
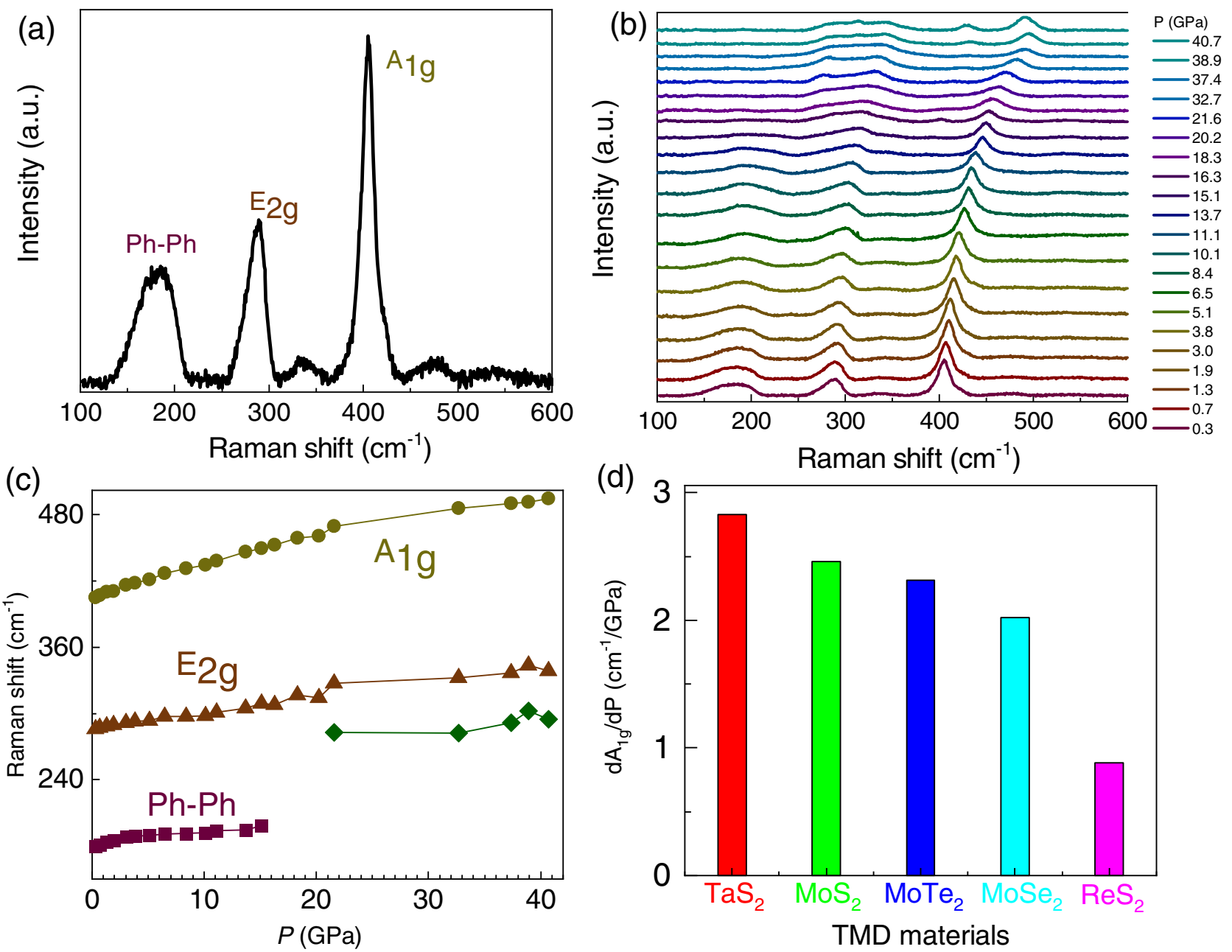

FIG. 1. (a) Raman scattering spectra of $2 \mathrm{H}-\mathrm{TaS}_{2}$ under room temperature and ambient pressure. (b) Raman of spectra under various hydrostatic pressures up to $40 \mathrm{GPa}$ at room temperature. (c) Pressure dependence of various vibrational modes in $2 \mathrm{H}-\mathrm{TaS}{ }_{2}$. $\mathrm{Ph}-\mathrm{Ph}$ refers to a two-phonon mode. (d) Comparison of the pressure coefficient $\left(d A_{1} g / d P\right)$ of the out-of-plane $\left(A_{1 g}\right) \operatorname{Raman}_{\text {peaks of TaS }}$, MoS $\mathrm{M}_{2}$ $\mathrm{MoSe}_{2}, \mathrm{MoTe}_{2}$, and $\mathrm{ReS}_{2}$ [35]. One can see that the out-of-plane mode of $\mathrm{TaS}_{2}$ presents the highest pressure coefficient among all selected materials. 

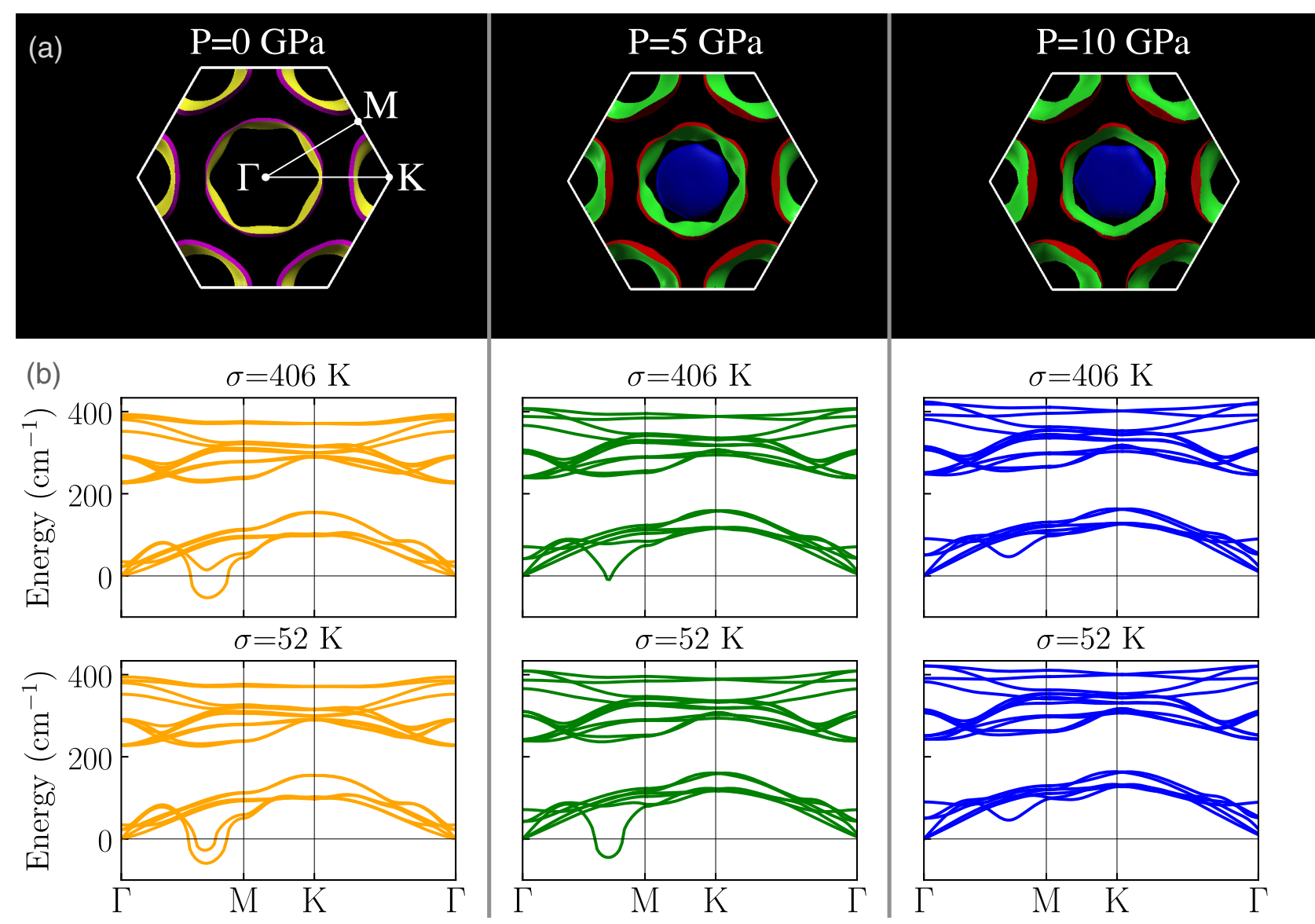

FIG. 2. Calculated Fermi surfaces (a) and phonon dispersions (b) for three different values of external pressure. The phonon dispersions were calculated for two different electronic temperatures, defined by the smearing parameter $\sigma$. The Fermi surface was plotted using XCrySDen software (see text for more details).

represents a two-phonon mode, is detectable up to 15.1 GPa, whereas other peaks exist up to our final pressure of $40 \mathrm{GPa}$. The temperature dependency of the two-phonon mode is similar at ambient and high pressure, and suppression of the two-phonon mode by reduction of temperature results in the appearance of the CDW mode [34]. So, a pressure-induced structural phase transition could be expected well above critical pressure of the two-phonon mode of $15.1 \mathrm{GPa}$. For the case of the $A_{1 g}$ mode, it shows a blueshift with a deformation coefficient of $2.83 \mathrm{~cm}^{-1} / \mathrm{GPa}$. Pressure coefficients of $A_{1 g}$ modes of similar TMDs are compared in a bar diagram [Fig. 1(d)]. As the $A_{1 g}$ mode is vibrationally coupled with the $E_{2 g}$ mode in the case of $\mathrm{TaS}_{2}$, the pressure coefficient of the $A_{1 g}$ mode of $2 \mathrm{H}-\mathrm{TaS}_{2}$ is higher than in any other TMD material. As the pressure increases, the pressure coefficient of $A_{1 g}$ is reduced above $20 \mathrm{GPa}$, at which bifurcation of the $E_{2 g}$ mode starts to occur. As noticed in $\mathrm{MoS}_{2}$ [35], this phenomenon might be due to the structural transition.

Recently, first-principles calculations have shown that the electron-phonon interactions depend on both the amount of applied strain and the direction in $2 \mathrm{H}-\mathrm{TaSe}_{2}$ [36]. In addition, a sudden change in the $E_{2 g}$ mode in
$2 \mathrm{H}-\mathrm{TaSe}_{2}$ is observed [28]. In order to get a physical insight into the suppression of the CDW phase under pressure, we have investigated the electronic structure by means of $a b$ initio theory. The main results are shown in Fig. 2. According to the results obtained for undistorted $2 \mathrm{H}-\mathrm{TaS}_{2}$, the material undergoes a pressure-induced electronic topological (so-called Lifshitz [37]) transition. An additional hole pocket around the $\Gamma$ point emerges, as shown in blue on Fig. 2(a). This transition happens below $2.5 \mathrm{GPa}$, and upon further compression, at least up to $15 \mathrm{GPa}$, the Fermi surface topology is intact, while its shape becomes slightly modified. The pressure evolution of the calculated phonon spectra is shown in Fig. 2(b). At the equilibrium, and under small applied pressures, there is a phonon instability along the $\Gamma-M$ direction at the wave vector close to experimental $q_{\mathrm{CDW}}$. Upon compression, the instability is suppressed somewhere between 5 and $10 \mathrm{GPa}$, indicating the suppression of the CDW order. Interestingly, the instability disappears after the ETT, which indicates that the Fermi surface nesting itself is not the only driving force of CDW order, which is in line with other, more recent studies on $2 \mathrm{H}-\mathrm{TaS}_{2}$ [27]. The results of our calculations for ambient pressure are in agreement with Ref. [33]. These 

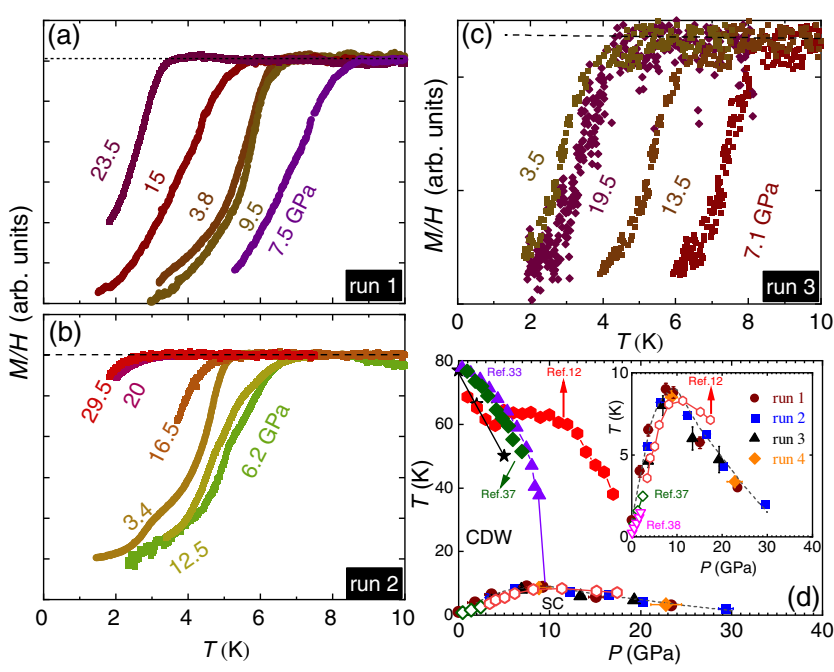

FIG. 3. (a)-(c) The temperature dependence of the dc susceptibility components of $2 \mathrm{H}-\mathrm{TaS}_{2}$ measured in dc field with an amplitude of $30 \mathrm{Oe}$ at elevated pressures in three runs. (d) The obtained pressure-temperature $(P-T)$ phase diagram of $2 \mathrm{H}-\mathrm{TaS}_{2}$. Pressure dependence of the SC transition temperatures $T_{c}$ up to $30 \mathrm{GPa}$. The values of $T_{c}$ were determined from the high-pressure resistivity and dc magnetic susceptibility $[11,29]$. The temperature dependence on the disappearance of the CDW as a function of pressure, is shown as stars and the data points from the work of Refs. $[12,32,39,40]$ were taken for comparison.

types of calculations are not able to properly capture the CDW transition temperature, but show a correct qualitative behavior. As the electronic temperature increases, the phonon instability becomes less pronounced, but persists up the temperatures well above experimental CDW ordering temperature. The authors of Ref. [33] attribute this to the presence of a short-range CDW state. We would like to note, however, that the employed treatment of the temperature effects is potentially oversimplified and does not capture many phenomena. The main reason is an incomplete description of the electronic correlations within DFT. Moreover, certain crystal structures are known to be stabilized due to anharmonic effects [38]. An explicit account of the electron-phonon interaction which is expected to be quite anisotropic [27] might induce strong modifications of both electronic and phononic spectra.

High-pressure magnetic measurements have repeated in four independent runs using different pieces of the same sample and the data are highly reproducible, Figs. 3(a)-3(c) [38]. The domelike evolution of $T_{c}$ was constructed based on the observed pressure-dependent magnetization data shown in Fig. 3(d). The data points from Refs. [12,32,39,40] were taken for comparison. Some notable differences with Ref. [12] can be seen. This discrepancy might result from the presence of a pseudogap as reported by ARPES measurements at ambient pressure above $T_{\mathrm{CDW}}$ and which may survive up to higher temperatures [41]. As displayed in Figs. 3(a)-3(c), it is clear that $T_{c}$ increased up to a pressure of $8.5 \mathrm{GPa}$, where it exhibits a maximum, then immediately begins to turn down. This kind of dome-shaped curve is one of the hallmarks of high temperature superconductors, but many mysteries around these types of domes remain to be explained [42]. Deriving a solid picture of the origin of the SC dome constitutes a major challenge. Applying external pressure to the system simply modifies the interatomic spacing, wave function overlap and electronic structure, as well as the balance between kinetic energy and Coulomb interaction among the electrons. The SC state certainly depends on these parameters, which is determined by both the pressure and the existence of the CDW state. Since both pressure and the CDW state heavily influence $T_{c}$, the competition between the two might be the cause of the SC dome.

Low-temperature ac susceptibility, $\chi$ ', measurements as illustrated in Fig. 4(a). $T_{c}$ of $1.2 \mathrm{~K}$ has been extracted from the bifurcation point between $\chi_{v}^{\prime}$ and $\chi_{v}^{\prime \prime}$. One can clearly see that the maximum of the imaginary part of ac susceptibility, see Fig. 4(a) (right inset), shifts to higher temperatures upon increasing the frequency which we attribute that to the motion of vortices. The lower critical field is a very useful parameter providing key information regarding bulk thermodynamic properties. The most popular approach of determining the $H_{c 1}$ compared with the values obtained from the onset of the trapped magnetic moment [ $\left(M_{t}\right.$ Fig. 4(c) ] [29]. We have confirmed the absence of the surface barriers in our case from the very symmetric dc magnetization hysteresis curves at $350 \mathrm{mK}$ [Fig. 4(a) (left inset)]. The experimental values of $H_{c 1}$ were corrected by accounting for the demagnetization effects and the absolute value can be estimated by using the relation proposed by Brandt [43]. The most intriguing feature in Fig. 4(d) is the upward trend with negative curvature over the entire temperature range, similar features are reported in Refs. [44,45]. The obtained experimental temperature dependence of $H_{c 1}$ shown in Fig. 4(d) was analyzed using the phenomenological $\alpha$ model $[29,46]$. The main features from the corrected $H_{c 1}$ values in Fig. 4(d) can be described in the following way: (i) As a first step we compare our data to the single band $s$ wave and we find a systematic deviation at high temperature data; (ii) more obvious deviations exist in the case of $d$-wave approach [46]. This clearly indicates that the gap structure of our system is more likely to be nodeless $s$ wave, and (iii) then, the anisotropic $s$ wave is further introduced to fit the experimental data. For the anisotropic $s$ wave, the fitting with the magnitude of the gap $\Delta_{0}=1.21 \mathrm{meV}$ with an anisotropy parameter $\approx 1.01$. As can be seen the anisotropic s-wave order parameter presents a good description to the data. We hence conclude that in $\mathrm{Ta} S_{2}$ the exotic SC gap structure is related to the Ta tubular sheets and that, even if the charge density wave is perturbing those sheets in $\mathrm{TaS}_{2}$, this CDW does not affect the SC gap structure. 

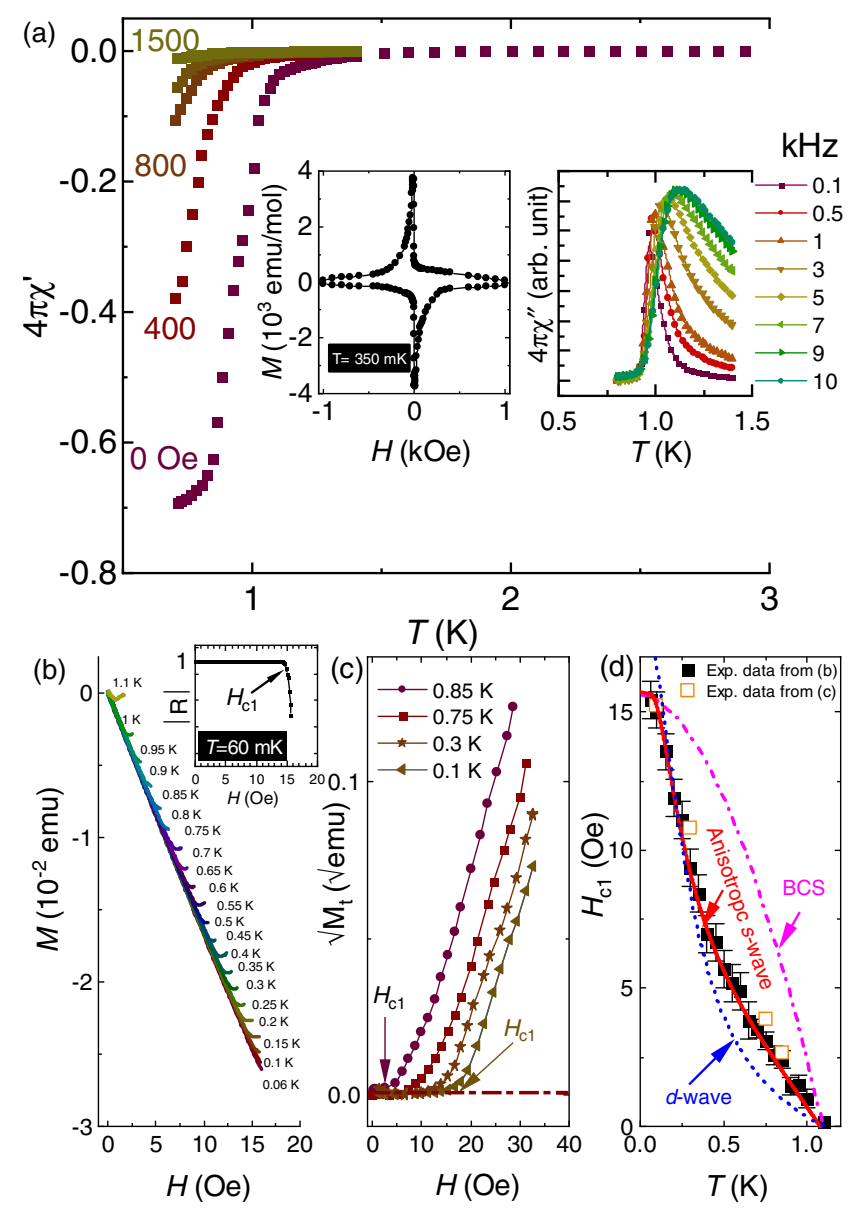

FIG. 4. (a) The temperature dependence of the complex ac susceptibility $4 \pi \chi_{v}^{\prime}$ measured in an ac field with an amplitude of 5 Oe and a frequency of $1 \mathrm{kHz}$. The insets illustrate the magnetization $M$ vs $H$ loops measured at $350 \mathrm{mK}$ and the imaginary part of ac at various frequency $\left(\nu_{m}\right)$. (b) The SC initial part of the magnetization curves measured at various temperatures down to $60 \mathrm{mK}$. The inset depicts an example used to determine the $H_{c 1}$ value using the regression factor $R$, at $T=60 \mathrm{mK}$ [29]. (c) The field dependence of $\sqrt{M_{t}}$ at various temperatures. The arrows indicate $H_{c 1}$ values that estimated by extrapolating the linear fit of $\sqrt{M_{t}}$ to 0 . (d) Phase diagram of $H_{c 1}$ for the field applied parallel to the $c$ axis. The solid red line is the fitting curves using anisotropic $s$-wave approach. The dotted and dashed lines represent the $d$ wave and a single-gap BCS approach, respectively.

The temperature-pressure phase diagram of $\mathrm{TaS}_{2}$ is demonstrated here to have a domelike SC phase with a maximum $\mathrm{SC}$ transition temperature $T_{c}=9.1 \mathrm{~K}$. By employing $a b$ initio electronic structure theory, we were able to investigate the temperature and pressure dependence of the phonon spectrum. It is shown that, at ambient conditions, there is a phonon instability at the propagation vector close to the $q_{\mathrm{CDW}}$ wave vector. Furthermore, the temperature dependence measurements of the critical field are consistent with single gap anisotropic $s$-wave superconductivity. The role of the two-phonon mode on the
CDW transition, which was highlighted in prior studies [34] needs to be better understood and calls for additional low-temperature Raman measurements.

Y. K. and M. A. H. acknowledge the financial support from the Swedish Research Council (VR) under the Projects No. 2019-03569 and No. 2018-05393. O.E. acknowledges support from the Swedish Research Council, the Knut and Alice Wallenberg Foundation and The Swedish e-science effort, eSSENCE. This work has been supported by Act 211 of the Government of Russian Federation, contracts 02.A03.21.0006 and 02.A03.21.0011. DFG through the Würzburg-Dresden Cluster of Excellence (EXC 2147, Project No. 39085490).

*mhafiez@g.harvard.edu

[1] T. Yokoya, T. Kiss, A. Chainani, S. Shin, M. Nohara, and H. Takagi, Science 294, 2518 (2001).

[2] M. B. Maple, Appl. Phys. 9, 179 (1976).

[3] P. C. Canfield, P. L. Gammel, and D. J. Bishop, Phys. Today 51, No. 10, 40 (1998).

[4] M. D. Johannes and I. I. Mazin, Phys. Rev. B 77, 165135 (2008).

[5] J. J. Hamlin, D. A. Zocco, T. A. Sayles, M. B. Maple, J. H. Chu, and I. R. Fisher, Phys. Rev. Lett. 102, 177002 (2009).

[6] D. A. Zocco, J. J. Hamlin, K. Grube, J. H. Chu, H. H. Kuo, I. R. Fisher, and M. B. Maple, Phys. Rev. B 91, 205114 (2015).

[7] B. Sipos, A. F. Kusmartseva, A. Akrap, H. Berger, L. Foro, and E. Tutis, Nat. Mater. 7, 960 (2008).

[8] Q. H. Wang, K. Kalantar-Zadeh, A. Kis, J. N. Coleman, and M. S. Strano, Nat. Nanotechnol. 7, 699 (2012).

[9] A. K. Geim and I. V. Grigorieva, Nature (London) 499, 419 (2013).

[10] A. Majumdar, D. VanGennep, J. Brisbois, D. Chareev, A. V. Sadakov, A. S. Usoltsev, M. Mito, A. V. Silhanek, T. Sarkar, A. Hassan, O. Karis, R. Ahuja, and M. Abdel-Hafiez, Phys. Rev. Mater. 4, 084005 (2020).

[11] M. Abdel-Hafiez, X.-M. Zhao, A. A. Kordyuk, Y.-W. Fang, B. Pan, Z. He, C.-G. Duan, J. Zhao, and X.-J. Chen, Sci. Rep. 6, 31824 (2016).

[12] D. C. Freitas et al., Phys. Rev. B 93, 184512 (2016).

[13] K. E. Wagner, E. Morosan, Y.S. Hor, J. Tao, Y. Zhu, T. Sanders, T. M. McQueen, H. W. Zandbergen, A. J. Williams, D. V. West, and R. J. Cava, Phys. Rev. B 78, 104520 (2008).

[14] Y. Yu, F. Yang, X. F. Lu, Y. J. Yan, Y.-H. Cho, L. Ma, X. Niu, S. Kim, Y.-W. Son, D. Feng, S. Li, S.-W. Cheong, X. H. Chen, and Y. Zhang, Nat. Nanotechnol. 10, 270 (2015).

[15] C. Berthier, P. Molinie, and D. Jerome, Solid State Commun. 18, 1393 (1976).

[16] Z.-H. Chi, X.-M. Zhao, H. Zhang, A. F. Goncharov, S. S. Lobanov, T. Kagayama, M. Sakata, and X.-J. Chen, Phys. Rev. Lett. 113, 036802 (2014).

[17] B. Sipos, A. F. Kusmartseva, A. Akrap, H. Berger, L. Forro, and E. Tutis, Nat. Mater. 7, 960 (2008).

[18] X. Zhu, J. Guo, J. Zhang, and E. W. Plummer, Adv. Phys. X 2, 622 (2017). 
[19] J. A. Wilson, F. J. Di Salvo, and S. Mahajan, Phys. Rev. Lett. 32, 882 (1974).

[20] T. M. Rice and G. K. Scott, Phys. Rev. Lett. 35, 120 (1975).

[21] J. van Wezel, P. Nahai-Williamson, and S. S. Saxena, Phys. Rev. B 81, 165109 (2010).

[22] A. H. Castro Neto, Phys. Rev. Lett. 86, 4382 (2001).

[23] F. Weber, S. Rosenkranz, J.-P. Castellan, R. Osborn, R. Hott, R. Heid, K.-P. Bohnen, T. Egami, A. H. Said, and D. Reznik, Phys. Rev. Lett. 107, 107403 (2011).

[24] M. D. Johannes, I. I. Mazin, and C. A. Howells, Phys. Rev. B 73, 205102 (2006).

[25] M. D. Johannes and I. I. Mazin, Phys. Rev. B 77, 165135 (2008).

[26] L. P. Gor'kov, Phys. Rev. B 85, 165142 (2012).

[27] K. Wijayaratne, J. Zhao, C. Malliakas, D. Young Chung, M. G. Kanatzidis, and U. Chatterjee, J. Mater. Chem. C 5, 11310 (2017).

[28] H. M. Hill, S. Chowdhury, J. R. Simpson, A. F. Rigosi, D. B. Newell, H. Berger, F. Tavazza, and A. R. Hight Walker, Phys. Rev. B 99, 174110 (2019).

[29] See Supplemental Material at http://link.aps.org/ supplemental/10.1103/PhysRevLett.125.186401 for the experimental and calculation details and the supporting results.

[30] M. Abdel-Hafiez, M. Mito, K. Shibayama, S. Takagi, M. Ishizuka, A. N. Vasiliev, C. Krellner, and H. K. Mao, Phys. Rev. B 98, 094504 (2018).

[31] M. Abdel-Hafiez, Y. Zhao, Z. Huang, C.-w. Cho, C. H. Wong, A. Hassen, M. Ohkuma, Y. W. Fang, B. J. Pan, Z. A. Ren, A. Sadakov, A. Usoltsev, V. Pudalov, M. Mito, R. Lortz, C. Krellner, and W. Yang, Phys. Rev. B 97, 134508 (2018).

[32] R. Grasset, Y. Gallais, A. Sacuto, M. Cazayous, S. Manas-Valero, E. Coronado, and M.-A. Measson, Phys. Rev. Lett. 122, 127001 (2019).
[33] J. Joshi, H. M. Hill, S. Chowdhury, C. D. Malliakas, F. Tavazza, U. Chatterjee, A. R. Hight Walker, and P. M. Vora, Phys. Rev. B 99, 245144 (2019).

[34] X.-M. Zhao, K. Zhang, Z.-Y. Cao, Z.-W. Zhao, V. V. Struzhkin, A. F. Goncharov, H.-K. Wang, A. G. Gavriliuk, H.-K. Mao, and X.-J. Chen, Phys. Rev. B 101, 134506 (2020).

[35] S. Tongay, H. Sahin, C. Ko et al., Nat. Commun. 5, 3252 (2014).

[36] S. Chowdhury, J. R. Simpson, T. L. Einstein, and A. R. Hight Walker, Phys. Rev. Mater. 3, 084004 (2019).

[37] I. M. Lifshitz, Sov. Phys. JETP 11, 1130 (1960), http://www .jetp.ac.ru/cgi-bin/e/index/e/11/5/p1130?a=list.

[38] P. Souvatzis, O. Eriksson, M. I. Katsnelson, and S. P. Rudin, Phys. Rev. Lett. 100, 095901 (2008).

[39] R. Delaplace, P. Molinie, and D. Jerome, J. Phys. Lett. 37, 13 (1976).

[40] T. F. Smith, R. N. Shelton, and R. E. Schwall, J. Phys. F 5, 1713 (1975).

[41] J. Zhao, K. Wijayaratne, A. Butler, J. Yang, C. D. Malliakas, D. Y. Chung, D. Louca, M. G. Kanatzidis, J. van Wezel, and U. Chatterjee, Phys. Rev. B 96, 125103 (2017).

[42] J. Lu, O. Zheliuk, Q. Chen, I. Leermakers, N. E. Hussey, U. Zeitler, and J. Ye, Proc. Natl. Acad. Sci. U.S.A. 115, 3551 (2018).

[43] E. H. Brandt, Phys. Rev. B 60, 11939 (1999).

[44] C. Ren, Z.-S. Wang, H.-Q. Luo, H. Yang, L. Shan, and H.-H. Wen, Phys. Rev. Lett. 101, 257006 (2008).

[45] C. S. Yadav and P. L. Paulose, New J. Phys. 11, 103046 (2009).

[46] A. Carrington and F. Manzano, Physica (Amsterdam) 385C, 205 (2003). 